\title{
Intra-patient variability in tacrolimus trough levels over 2 years affects long-term allograft outcomes of kidney transplantation
}

\author{
Yohan Park ${ }^{1}$, Hanbi Lee ${ }^{2}$, Sang Hun Eum², Hyung Duk Kim² ${ }^{2}$ Eun Jeong Ko${ }^{2}$, Chul Woo Yang ${ }^{2}$, Byung Ha Chung ${ }^{2}$ \\ ${ }^{1}$ Department of Internal Medicine-Nephrology, Konyang University Hospital, Daejeon, Korea \\ ${ }^{2}$ Department of Internal Medicine-Nephrology, The Catholic University of Korea, Seoul St. Mary's Hospital, Seoul, Korea
}

Background: The current study aimed to determine the impact of tacrolimus (TAC) trough level (C0) intra-patient variability (IPV) over 2 years after kidney transplantation (KT) on allograft outcomes.

Methods: In total, 1,143 patients with low immunologic risk were enrolled. The time-weighted coefficient variability (TWCV) of TAC-CO was calculated, and patients were divided into tertile groups (T1: $<24.6 \%, \mathrm{~T} 2: 24.6 \%-33.7 \%, \mathrm{~T} 3: \geq 33.7 \%$ ) according to TAC-C0-TWCV until post-transplant 1st year. Moreover, they were classified into the low/low, low/high, high/low, and high/high groups based on a TAC-C0-TWCV value of 33.7\% during post-transplant $0-1$ st and 1st-2nd years. We compared the allograft outcomes among the three tertile and four TAC-CO-TWCV groups.

Results: The T3 group had the highest rate of death-censored allograft loss (DCGL), and T3 itself was an independent risk factor for DCGL (adjusted hazard ratio [HR], 1.853; $P=0.029$ ). In addition, sustained TWCV $\geq 33.7 \%$ until 2 years after $K T$ showed the highest risk for DCGL (HR, 2.395; $P=0.013)$. Moreover, the changes in TWCV during the 1st-2nd post-transplant year significantly affect to DCGL occurrence (HR of low/high 2.086, $\mathrm{P}=0.045$; HR of high/low 1.813, $\mathrm{P}=0.021$ ). Patients with an average TAC-C0 of $\geq 5 \mathrm{ng} / \mathrm{mL}$ in the high/high group were at highest risk for DCGL as well.

Conclusions: TAC-IPV is an important factor that can significantly affect comprehensive allograft outcomes. TAC-IPV after 1st year of KT was also considered an important factor for allograft outcomes. Moreover, TAC-IPV can significantly affect allograft outcomes even with a high average TAC-CO.

\footnotetext{
(C) The Korean Society for Transplantation

This is an Open Access article distributed under the terms of the Creative Commons Attribution Non-Commercial License (http://creativecommons.org/licenses/by-nc/4.0/) which permits unrestricted non-commercial use, distribution, and reproduction in any medium, provided the original work is properly cited.
} 